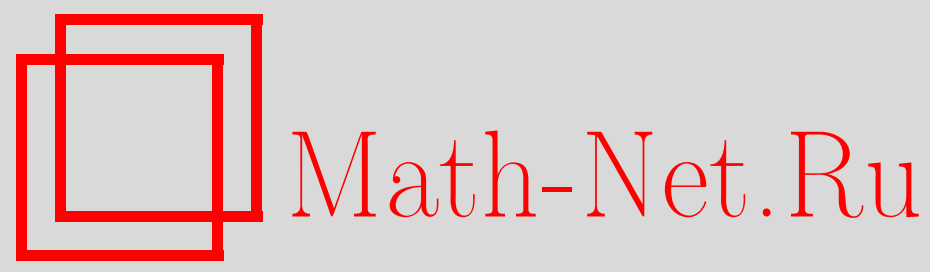

С. И. Окрут, Обобщенные теоремы Хермана и голоморфные конформные субмерсии, Матем. заметки, 1999, том 66, выпуск 1, 120-134

DOI: https://doi.org/10.4213/mzm1147

Использование Общероссийского математического портала Math-Net.Ru подразумевает, что вы прочитали и согласны с пользовательским соглашением http://www.mathnet.ru/rus/agreement

Параметры загрузки:

IP : 54.166 .219 .16

26 апреля 2023 г., 13:46:41

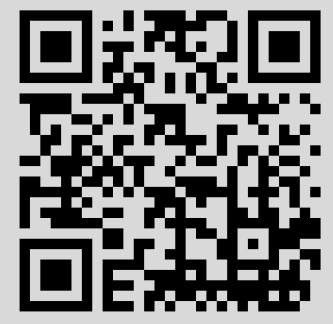




\title{
ОБОБЩЕННЫЕ ТЕОРЕМЫ ХЕРМАНА И ГОЛОМОРФНЫЕ КОНФОРМНЫЕ СУБМЕРСИИ
}

\section{С. И. Окрут}

\begin{abstract}
В статье показано, что если некоторое кэлерово многообразие допускает голоморфную риманову субмерсию, то это многообразие является локально приводимым. Получено обобщение известных теорем Р. Хермана на случай конформных и голоморфных конформных субмерсий. Предложен метод конструирования кэлеровых расслоенных пространств с голоморфной конформной (неримановой) проекцией и со вполне геодезическими изоморфными слоями. Метод допускает построение полных, в том числе компактых, кэлеровых расслоенных пространств указанного типа.
\end{abstract}

Библиография: 10 названий.

Введение. В статье [1] Р. Херманом был решен вопрос о том, при каких достаточных условиях риманова субмерсия является проекцией расслоения с изометричными между собой слоями. Как показоно в теореме 1, если кэлерово многообразие допускает голоморфную риманову субмерсию, то оно необходимо локально приводимое. Однако неприводимые кэлеровы многообразия допускают голоморфные конформные субмерсии (определение 1 и примеры 1, 2). Цель этой статьи ответить на вопрос при каких достаточных условиях голоморфная конформная субмерсия кэлерова многообразия является проекцией голоморфного расслоения с изоморфными между собой слоями. Ответ на этот вопрос дается в теоремах 3,4 . Теоремы 5,6 предоставляют эффективный способ построения расслоений указанного типа со вполне геодезическими слоями, в том числе полных. Изучение вводимых ниже (определение 2) скрещенных кэлеровых расслоений представляет интерес прежде всего в связи с возможностью строить кэлеровы аналоги скрешенных произведений [2].

Произвольно выбираемые многообразия, функции и тензорные поля предполагаются гладкими (класса $C^{\infty}$ ). А основные понятия и обозначения соответствуют принятым (см., например, [3], [4]). Кроме того, будем предполагать, что все произвольно выбираемые многообразия являются связными.

1. Голоморфные и римановы субмерсии. Для всякой субмерсии $\nu$ риманова многообразия $E$ на риманово многообразие $M$ в каждой точке $\Xi \in E$ имеется прямое ортогональное разложение касательного пространства $T_{\Xi} E=V_{\Xi}+H_{\Xi}$ на вертикальное и горизонтальное подпространства. Субмерсия называется плоской, если ее горизонтальное распределение $H$ вполне интегрируемое; получаемое в этом случае слоение назьвается горизонтальным. Символы $V$ и $H$ будут одновременно служить для обозначения операторов ортогонального проектирования на одноименно обозначенные распределения. Операторы $V$ и $H$ стандартным образом (см. [3, гл. 1, предложение 2.13]) 
продолжаются на всю алгебру тензоров в каждой точке многообразия $E$. И тензор $K$, в том числе вектор или форма, будет назьваться вертикальным (горизонтальным), если его горизонтальная (вертикальная) компонента равна нулю $H K=0$ ( $V K=0$ соответственно). Далее всюду в статье вертикальные векторные поля субмерсии обозначаются через $U, V, W$, а базисные, т.е. проектируемые и горизонтальные, - через $X$, $Y, Z$. Локальная проектируемость векторного поля $P$ на базу эквивалентна следующему свойству:

$$
L_{U} P=[U, P] \in V \text {. }
$$

Голоморфность субмерсии немедленно влечет инвариантность относительно комплексной структуры вертикального распределения, а эрмитовость метрики $g$ на $E$ (т.е. $g(J P, Q)=-g(P, J Q))$ - инвариантность горизонтального распределения. Имеют место равенства

$$
\nu_{*} J=J \nu_{*}, \quad J V=V, \quad J H=H .
$$

ЛЕмма 1. Пусть $\nu$ голоморфная субмерсия кәлеровых многообразий. Субмерсия $\nu$ является римановой тогда и только тогда, когда она является плоской.

ДокАЗАтЕльство. Пусть $X, Y$ - базисные векторные поля, а $W$ вертикальное векторное поле. Применим свойство замкнутости кэлеровой формы $\Phi$ многообразия $E \mathrm{k}$ следующей тройке полей $d \Phi(W, X, Y)=0$. Из свойства проектируемости (1.1) следует формула:

$$
W \Phi(X, Y)+\Phi(W,[X, Y])=0 .
$$

Если воспользоваться взаимосвязью между кэлеровой формой и кэлеровой метрикой, то из формулы (1.3) для голоморфной субмерсии следует равенство

$$
\left(L_{W} g\right)(X, J Y)=\Phi([X, Y], W)
$$

Критерием того, что субмерсия является римановой, служит равенство нулю левой части равенства (1.4) для любых горизонтальных векторных полей $X$ и $Y$. Теперь теорема следует из равенства (1.4) и теоремы $Ф$ робениуса.

ТЕорема 1. Если голоморфная плоская субмерсия $\nu$ со вполне геодезическими слоями определена на кэлеровом многообразии $E$, то кәлерово многообразие $E$ локально приводимое.

ДоКАЗАТЕЛЬСТво. Из леммы 1 следует, что субмерсия $\nu$ - римановая. На основании равенств (1.2) слои полученного горизонтального слоения $\mathcal{H}$ субмерсии $\nu$ являются комплексными подмногообразиями. Порожденная слоением локальная субмерсия $\chi_{\text {loc }}$ вдоль слоев $\mathcal{H}$ на локальньй фактор (см. [5, с. 15]) $E / \mathcal{H}$ также является голоморфной. Из двойственности [6, с.60] между римановыми и геодезическими слоениями следует локальная приводимость метрики $E$.

\section{2. Когда голоморфная конформная субмерсия является проекцией рас- слоения.}


ОПРЕДЕЛЕНИЕ 1. Пусть имеется субмерсия $\nu$ из $E$ на $M$ и пусть $g$ и $g_{M}$ римановы метрические тензоры в $E$ и $M$ соответственно. Субмерсия $\nu$ из $E$ на $M$ назьвается конформной, если существует функция $f$ на $E$ такая, что $H g=\exp (2 f) \nu^{*} g_{M}$.

При этом $f$ назьвается вертикальным показателем конформности субмерсии, если $f$ вертикальная функция, т.е. $X f=0 \forall X$-горизонтального вектора. Риманова субмерсия получается как частный случай конформной субмерсии при $f \equiv 0$. Будем в дальнейшем обозначать градиент функции через $\operatorname{gr} f, \operatorname{agr}^{c} f=J \operatorname{gr} f$, где $J$ - это почти комплексная структура. Следующая теорема 2 является обобщением известной теоремы Р. Хермана [4, пункт 9.42].

ТЕорема 2. Если $\nu: E \mapsto M-$ конформная субмерсия полного риманова многообразия Е с вертикальным показателем конформности, то $\nu$ является проекиией локально тривиального расслоения $\nu$, база которого также полное риманово многообразие.

ДокАЗАТЕЛЬСТво. Пусть $X^{H}$ горизонтальный лифт векторного поля $X$ базы. Из теоремы 3.2(i) и формулы (3.9), приведенных в [7], следует равенство

$$
\nabla_{X^{H}} X^{H}=\left(\nabla_{X} X\right)^{H}-\left\|X^{H}\right\|^{2} \operatorname{gr} f .
$$

Пусть $\gamma=x_{s}(|s|<\varepsilon)$ геодезическая базы и $s$ ее натуральньй параметр. Через $X=$ $\left\{X_{s}=\dot{x}_{s}\right\}$ обозначим векторное поле скорости $\|X\|=1$. Интегральные кривые $\Xi_{s}$ горизонтального лифта $X^{H}$ проектируются в интегральныекривыеполя $X$, т.е. $\nu \Xi_{s}=x_{s}$. Рассмотрим систему уравнений для кривых $\Xi_{s}$ в $E$ :

$$
\begin{gathered}
\nabla_{Z} Z+\|Z\|^{2} \operatorname{gr} f=0 \\
Z=\dot{\Xi}_{s} .
\end{gathered}
$$

На основании теоремы существования и единственности решения задачи Коши для обькновенных дифференциальных уравнений и формулы (2.1) решение $\Xi_{s}$ с такими начальными условиями, что $Z_{0}=\dot{\Xi}_{0}-$ это горизонтальньй вектор, является горизональной кривой, проектирующейся на геодезическую базы. Вычислим

$$
\frac{d}{d s}\|Z\|^{2}=2 g\left(\nabla_{Z} Z, Z\right)=-2\|Z\|^{2} d f(Z)=0
$$

при условии, что показатель конформности - вертикальная функция. Таким образом, норма вектора скорости величина постоянная вдоль любой интегральной кривой системы (2.2), (2.3).

Покажем, что всякая интегральная кривая $\Xi_{s}(0 \leqslant s<\varepsilon)$ продолжается в точку $s=\varepsilon$. Рассмотрим последовательность $s_{n} \rightarrow \varepsilon$ при $n \rightarrow \infty$. Обозначим $\Xi_{n}=\Xi_{s_{n}}$. Имеем

$$
\operatorname{dist}\left(\Xi_{m}, \Xi_{n}\right)=\left|\int_{s_{m}}^{s_{n}}\|Z\| d s\right|=e^{f\left(\Xi_{0}\right)}\left|s_{n}-s_{m}\right| .
$$

Поэтому последовательность $\Xi_{n}$ является фундаментальной. Из полноты $E$ следует, что существует $\Xi=\lim _{n \rightarrow \infty} \Xi_{n}$. Очевидно, $\Xi$ не зависит от выбора последовательности $s_{n}$. Поэтому $\Xi=\lim _{s \rightarrow \varepsilon} \Xi_{s}$. Отсюда, во-первых, следует, что геодезическая 
базы $x_{s}$ может быть продолжена в точку $x=\lim _{s \rightarrow \varepsilon} x_{s}$. По теореме Хопфа-Ринова база $M$ является полным римановым многообразием. Горизонтальный лифт геодезической, продолженной за точку $s=\varepsilon$, с началом в точке $\Xi$ позволяет получить продолжение интегральной кривой $\Xi_{s}$ за точку $s=\varepsilon$; здесь снова используется теорема о единственности решения задачи Коши для (2.2)-(2.3). Таким образом, интегральные кривые системы уравнений $(2.2),(2.3)$ бесконечно продолжаемые. А из связности сегмента следует существование и единственность горизонтального лифта любой геодезической, соединяющей пару точек базы. Так как любую кривую базы можно аппроксимировать геодезическими дугами, то горизонтальное распределение субмерсии $\nu$ является связностью Эресмана [4, с.337]. По теореме Эресмана [4, пункт 9.40] $\nu$ является на самом деле проекцией локально тривиального расслоения.

СлЕДСТВИЕ 1. В условиях теоремы 2 горизонтальное распределение субмерсии является связностью Эресмана.

ЗАмЕчАнИЕ. Из сравнения результата Номидзу-Одзеки [8] и теоремы 2 следует, что условие вертикальности показателя конформности в теореме 2 является существенным.

Говоря, $E$ является расслоением со стандартным слоем $S$, когда $S$ является $p u м a-$ новым (а в частности, и кэлеровым) многообразием, будем подразумевать, что у каждой точки базы $M$ имеется локальная тривиализация $(O, \sigma)$ такая, что сужение $\sigma$ на каждьй слой является римановой изометрией слоя (относительно индуцированной из $E$ метрики) на стандартньй слой $S$. Это же обстоятельство будем выражать, называя $E$ расслоением с изометричнымимежду собой слоями. Естественно, все слои расслоения при этом изометричны стандартному слою $S$.

Лемма 2. Пусть у субмерсия риманова многообразия Е со вполне геодезическими слоями. Если горизонтальное распределение является связностью Эресмана, то Е-расслоение с изометричными между собой слоями.

ДокАЗАТЕЛЬСТво. Из теоремы Эресмана [4, пункт 9.40], следует, что $E$ является локально тривиальным расслоением. Предложение 3.3 из [1] означает, что диффеоморфизм слоев $\tau_{\gamma}: F_{a} \mapsto F_{b}$ из доказательства Эресмана в случае вполне геодезичности слоев является изометрией.

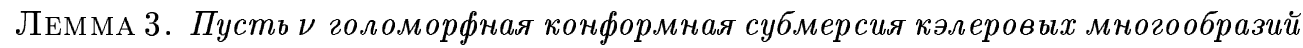
из Е на $M$ с вертикальным показателем конформности $f$ и вполне геодезическими слоями. Тогда

$$
A_{X} U=\nabla_{U} X=\omega(U) X+\omega^{c}(U) J X,
$$

где А инвариант О'Нейла субмерсии $\nu$, а вертикальные 1-формы $\omega, \omega^{c}$ определяются $\operatorname{ma\kappa }: \omega=d f, \omega^{c}=J \omega$.

ДокАЗАТЕЛьство. Пусть $X, Y$ - базисные векторные поля, а $U$ вертикальное векторное поле. Применим свойство замкнутости кэлеровой формы $\Phi$ многообразия $E \mathrm{k}$ следующей тройке полей $d \Phi(J U, X, J Y)=0$. Отсюда следует, что

$$
J U \Phi(X, J Y)+\Phi(J U,[X, J Y])=0 .
$$

Тогда

$$
2 \Phi\left(\nabla_{U} X, Y\right)=U \Phi(X, Y)+J U \Phi(X, J Y)
$$


Формула (2.7) следует из формулы (2.6) и выражения $2 \Phi\left(\nabla_{U} X, Y\right)=U \Phi(X, Y)-\Phi(J U$, $[X, J Y])$ для ковариантной производной, которая, как известно, является ковариантной производной связности Леви-Чивита. Снова используя связь между кэлеровой метрикой и ассоциированной с ней кэлеровой формой $\Phi(X, Y)=g(X, J Y)$, а также конформность субмерсии, определение 1 , получим равенство

$$
\left(L_{U} \Phi\right)(X, Y)=2 \omega(U) \Phi(X, Y)
$$

Свойство проектируемости (1.1) полей $X, Y$ влечет, что $L_{U} \Phi(X, Y)=U \Phi(X, Y)$. Поэтому, подставляя в формулу (2.7) выражение вида $(2.8)$, получим равенство

$$
\Phi\left(\nabla_{U} X, Y\right)=\omega(U) \Phi(X, Y)+\omega(J U) \Phi(X, J Y)
$$

Из эрмитовости кэлеровой формы $\Phi(X, J Y)=-\Phi(J X, Y)$, ееневырожденности и определения результата действия почти комплексной структуры на 1-форму, т.е. $J \omega(U)=-\omega(J U)$, следует $H \nabla_{U} X=\omega(U) X+\omega^{c}(U) J X$. Вполне геодезичность слоев влечет равенство $H \nabla_{U} X=\nabla_{U} X$, и второе равенство леммы доказано. Первое равенство леммы теперь следует из определения инварианта О’Нейла $A[4$, п. 9.20].

Далее будем считать, что все произвольно выбираемые расслоенные пространства являются локально тривиальньми. Локальной тривиализацией расслоенного пространства $E$ со стандартньм слоем $S$ и проекцией $\nu$ называется [4, пункт 9.47] пара $(O, \sigma)$ : окрестность базы $O$ и отображение $\sigma$, диффеоморфно отображающее порцию $\nu^{-1}(O)$ на $O \times S$. Если расслоенныепространства наделены римановой метрикой, то конформным расслоением будет назьваться расслоенние, проекция которого является конформной субмерсией. Учитьвая результаты п. 1, среди голоморфных конформньх субмерсий будем далее рассматирвать лишь такие, показатель конформности которых не является постоянньм.

Расслоение принято назьвать голоморфным, если: 1) как тотальное пространство, так и база расслоения являются комплексными многообразиями, а проекция $\nu$ - голоморфным отображением; 2) каждая точка должна обладать окрестностью $O$ и локальной тривиализацией $(O, \sigma)$ такой, что $\sigma$ является голоморфным отображением. Наконец, голоморфное расслоение будет назьваться кәлеровым расслоением, если база и тотальное пространство расслоения являются кэлеровыми многообразиями. Будем говорить, что кэлерово расслоение имеет изоморфные между собой слои, если имеется атлас локальных тривиализаций, для которого сужение тривиализующих отображений $\sigma$ на слой есть изоморфизм (т.е. голоморфная изометрия) кэлерового многообразия $\nu^{-1}(z)$ на стандартньй слой $S$.

ОПРЕДЕЛЕНИЕ 2. Кэлерово конформное расслоение с вертикальньм (непостоянным) показателем конформности и со вполне геодезическими и изоморфными между собой слоями будет нызываться скрещенным кәлеровым расслоением.

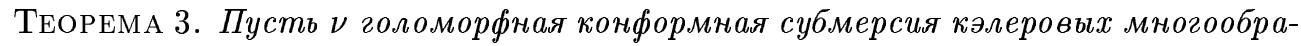
зий со вполне геодезическими слоями и вертикальным показателем конформности. Если горизонтальное распределение является связностью Эресмана, то $E$ скрещенное кэлерово расслоение. 
ДокАЗАТЕЛЬСТво. Учитьвая лемму 2, достаточно показать, что изометрия слоев $\tau_{\gamma}$ является голоморфным отображением. Для этого достаточно показать, что $\left(L_{Z} J\right) U=0$, где $Z$ - горизонтальньй лифт вектора скорости геодезической $\gamma$ из формулы (2.3). Так как $\left(L_{Z} J\right) U=J \nabla_{U} Z-\nabla_{J U} Z$, применив лемму 3 , получим

$$
\left(L_{Z} J\right) U=\omega(U) J Z-\omega^{c}(U) Z-\omega(J U) Z-\omega^{c}(J U) J Z=0 .
$$

Теорема 4. Пусть $\nu: E \mapsto M$ голоморфная конформная субмерсия кәлеровых многообразий с вертикальным показателем конформности и со вполне геодезическими слоями. Если Е полное многообразие, то Е-скрещенное кәлерово расслоение.

ДокАЗАТЕЛЬСтво. Требуемое утверждение есть комбинация следствия 1 и теоремы 3.

\section{3. Локальные примеры.}

ПримеР 1. Пусть кэлерова 2-форма $\Phi_{M}$, определенная на $M$, обладает кэлеровым потенциалом $F_{M}$, т.е. $\Phi_{M}=-2 i \partial \bar{\partial} F_{M}$. И пусть $y(t)$ вещественная функция, определенная на некотором вешественном интервале $(a, b)$ (допустимо и $a=-\infty, b=+\infty$ ), с отрицательной производной $\dot{y}(t)=\frac{d y}{d t}$. На комплексном многообразии $E$ из формулы (3.3) равенством (3.2) и формулой $f=y(t)$ определим пару функций $t$ и $f$. Тогда 2-форма $\Phi$

$$
\begin{gathered}
\Phi=e^{2 f}\left(\frac{2 i}{\pi} \dot{y}^{-1} \partial f \wedge \bar{\partial} f+\Phi_{M}\right), \\
\text { где } \quad t=\frac{1}{2}(\zeta+\bar{\zeta})-2 \pi F_{M},
\end{gathered}
$$

определенная на открытом множестве $E$,

$$
E=\left\{(\zeta, z) \in \mathbb{C} \times M \mid a<\frac{1}{2}(\zeta+\bar{\zeta})-2 \pi F_{M}(z, \bar{z})<b\right\},
$$

превращает $E$ в кэлерово многообразие. Действительно, так как $\Phi$ является формой типа $(1,1)$, ее инвариантность относительно почти комплексной структуры $J \Phi=\Phi$ очевидна. Покажем замкнутость этой формы. Имеем

$$
\begin{aligned}
d \Phi= & 2 e^{2 f} d f \wedge\left(\frac{2 i}{\pi} \dot{y}^{-1} \partial f \wedge \bar{\partial} f+\Phi_{M}\right) \\
& -\frac{2 i}{\pi} e^{2 f}\left(\dot{y}^{-3} \ddot{y} d f \wedge \partial f \wedge \bar{\partial} f-\dot{y}^{-1} \bar{\partial} \partial f \wedge \bar{\partial} f+\dot{y}^{-1} \partial f \wedge \partial \bar{\partial} f\right) \\
= & 2 e^{2 f} d f \wedge \Phi_{M}-\frac{2 i}{\pi} e^{2 f} \dot{y}^{-1} d f \wedge \partial \bar{\partial} f=2 e^{2 f} d f \wedge\left(\Phi_{M}+2 i \partial \bar{\partial} F_{M}\right)=0 .
\end{aligned}
$$

Здесь многократно использовалось разложение $d=\partial+\bar{\partial}$ и применялось следующее преобразование

$$
d f \wedge \partial \bar{\partial} f=\ddot{y} d f \wedge \partial t \wedge \bar{\partial} t+\dot{y} d f \wedge \partial \bar{\partial} t=-2 \pi \dot{y} d f \wedge \partial \bar{\partial} F_{M}
$$


Так как для любого вектора $Z$ типа $(1,0)$ выполняется $\bar{\partial} f(\bar{Z})=\partial f(Z)$, положительная определенность симметрической 2-формы $g(P, Q)=\Phi(J P, Q)$, ассоциированной с формой $\Phi$, очевидна в силу равенства

$$
g(Z, \bar{Z})=i \Phi(Z, \bar{Z})=e^{2 f}\left(-\frac{1}{\pi} \dot{y}^{-1}(Z f)^{2}+g_{M}(Z, \bar{Z})\right)
$$

и положительной определенности эрмитовой метрики базы, ассоциированной с $\Phi_{M}$. Покажем вполне геодезичность слоев построенного расслоения. Положим $W=\partial / \partial \zeta$ и $\bar{W}=\partial / \partial \bar{\zeta}$. Используя формулы $(9)-(12)$ из $[3, \S 5$, гл. IX], получим

$$
\nabla_{W} \bar{W}=0, \quad \nabla_{W} W=q W,
$$

где $q=-2 \pi\left(\dot{y}+\frac{1}{2} \ddot{y} \dot{y}^{-1}\right)$. А так как связность кэлерова, этим показано, что слои вполне геодезические.

Из способа задания кэлеровой формы $\Phi$ формулой (3.1) видно, что проекция $\nu$ на $M$ является конформной субмерсией с показателем конформности равным $f$. Пусть $\xi=$ $\operatorname{Re} \zeta$; несложно вычислить, что

$$
\operatorname{gr} f=-2 \pi \exp (-2 f) \frac{\partial}{\partial \xi}, \quad \operatorname{gr}^{c} f=-2 \pi \exp (-2 f) \frac{\partial}{\partial \eta},
$$

и поэтому показатель конформности является вертикальным. В итоге построено кэлерово многообразие $E$, допускающее голоморфную конформную субмерсию $\nu$ с вертикальным невырожденным показателем конформности и вполне геодезическими слоями. Построенное кэлерово многообразие $E$ является глобально тривиальньп скрещенным кэлеровьп расслоением над $M$ относительно проекции $\nu$ на $M$, стандартным слоем которого служит полоса $S:=\{\theta \in \mathbb{C} \mid a<\operatorname{Re} \theta<b\}$. В качестве тривиализации $(M, \sigma)$ можно выбрать голоморфное отображение $(z, \theta): E \mapsto M \times S$. Если кэлерова метрика в полосе $S$, стандартном слое, выбирается по формуле

$$
g_{S}=-\frac{1}{2 \pi} \exp (2 f(\operatorname{Re} \theta)) \dot{f} d \theta d \bar{\theta}
$$

то сужение тривиализации $(z, \theta): E \mapsto M \times S$, определенной формулой $\theta=t+i \operatorname{Im} \zeta$, на каждый слой является изоморфизмом кэлеровых многообразий.

ПРИмеР 2. Здесь будет построено семейство глобально тривиальных скрещенных кэлеровых расслоний $E$, но в этом примере, в отличие от примера 1 , показатель конформности будет иметь точки вырождения. Пусть, как и в примере $1,\left(M, \Phi_{M}\right)$ - кэлерово многообразие, а $F_{M}$ его кэлеров потенциал и пусть $l(s):[0 ; B) \mapsto \mathbb{R}$ гладкая (в нуле - справа), невырожденная (в том числе в нуле) функция. Кроме того, пусть вьполняется неравенство

$$
2 \lambda \int_{0}^{B} \sigma\left(\frac{d l}{d \sigma}\right)^{2} d \sigma<p_{0}^{2}
$$

в котором $\lambda$ некоторая вещественная константа. Определим гладкую положительную функцию $p(s)$ :

$$
\begin{gathered}
p^{2}(s)=p_{0}^{2}-2 \lambda \int_{0}^{s} \sigma\left(\frac{d l}{d \sigma}\right)^{2} d \sigma, \quad p_{0} \neq 0 \\
\frac{d p^{2}}{d l}=-2 \lambda s \frac{d l}{d s}
\end{gathered}
$$


Пусть

$$
E=\left\{(w, z) \in \mathbb{C} \times M|| w \mid \exp \left(-2 \pi \lambda F_{M}\right)<B\right\} .
$$

Многообразие $E$, определенное равенством (3.9), относительно проекции на $M$ является тривиальньм голоморфным расслоением. С помощью следующих формул определим на нем гладкую 2-форму $\Phi$ :

$$
\begin{gathered}
\Phi=-\frac{2 i}{\pi} \partial l(s) \wedge \bar{\partial} l(s)+p^{2}(s) \Phi_{M}, \\
\text { где } \quad s=|w| e^{-2 \pi \lambda F_{M}}: E \mapsto \mathbb{R} .
\end{gathered}
$$

Покажем, что если исключить из $E$ комплексную гиперповерхность $w=0$, на которой функция $p^{2}$ вырождается, и сделать разрез, исключив подмногообразие $\arg w=\pi$ вещественной коразмерности 1 , то получим расслоенное пространство из примера 1 . Вещественная функция $y$

$$
y=\ln p(s)
$$

после замены переменных по первой формуле из

$$
s=\exp (\lambda t), \quad w=\exp (\lambda \zeta)
$$

имеет отрицательную производную по $t$ на вещественном интервале $\left(-\infty ; \frac{1}{\lambda} \ln B\right)$. Это следует из формулы (3.7). По-прежнему будем обозначать точкой производную по $t$. Если теперь рассматривать $t$ как функцию на $E$, определенную формулой $(3.2)$ при замене голоморфных координат по второй формуле из (3.13), причем $|\operatorname{Im} \lambda \zeta|<\pi$, то для дифференциалов, определенных на $E$ из формул $(3.12),(3.7)$ и (3.8), получим (знаки \pm выбираются одинаковьми и равньми $\operatorname{sgn}(-\lambda d l / d s))$

$$
d l= \pm e^{f}(-\dot{y})^{-1 / 2} d f, \quad d^{c} l=J d l= \pm e^{f}(-\dot{y})^{-1 / 2} d^{c} f .
$$

А так как для любой функции $l$ на комплексном многообразии вьполняется равенство $2 i \partial l \wedge \bar{\partial} l=d l \wedge d^{c} l$, последние два равенства позволяют заключить, что форма $\Phi$ в равенствах (3.10) и (3.1) - это одна и та же форма. Таким образом, $E$ без гиперповерхности $w=0$ есть расслоенноепространство из примера 1. Точки исключенной гиперповерхности являются предельными точками открытого множества, которое, как теперь установлено, есть кэлерово многообразие. Проекция на $M$ является голоморфной конфомной субмерсией с вертикальным показателем конформности. А так как форма (3.10) гладко определена всюду на $E$, для того чтобы показать, что $E$ целиком является скрещенным кэлеровым расслоением, достаточно в силу соображений непрерьвности доказать лишь положительную определенность в точках гиперповерхности $w=0$ симметрической 2-формы $g$, ассоциированной с $\Phi$. Если обозначить через $g_{M}$ риманову метрику на $M$, ассоциированную с $\Phi_{M}$, то для $g$ получаем выражение

$$
\begin{gathered}
g=\frac{1}{2 \pi}\left(d l^{2}+d^{c} l^{2}\right)+p^{2}(s) g_{M}, \\
\left.g\right|_{w=0}=\frac{1}{2 \pi}\left(d l^{2}+d^{c} l^{2}\right)+p_{0}^{2} g_{M} .
\end{gathered}
$$


Так как $p_{0} \neq 0$, из формулы (3.16) следует положительная определенность $g$ в точках рассматриваемой гиперповерхности и конформность субмерсии. Кроме того, из выражения (3.15) становится прозрачньм геометрический смысл функции $l$ на $E$. С точностью до деления на константу $\sqrt{2 \pi}$ она является дистанцией между гиперповерхностями $l=$ const.

Точки гиперповерхности $w=0$ соответствуют точкам, где функция $s$ из (3.11) обращается в нуль. Из выражения $f$ через $l$, полученного с помощью равенств (3.12) и (3.8), следует равенство (3.17). А так как $f$ есть ничто иное, как показатель конформности, равенство

$$
d f=-\lambda s \frac{d l}{d s} p^{-2}(s) d l
$$

приводит к тому, что в точках рассматриваемой гиперповерхности $w=0$ имеется вырождение показателя конформности, т.е. $\left.d f\right|_{s=0}=0$.

4. Построение скрещенных кэлеровых расслоений “в целом". Многообра-

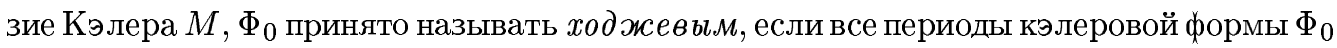
целократны некоторому вещественному числу. Это эквивалентно тому, что кэлерова форма пропорциональна $\Phi_{0}=r \Phi_{M}$ - некоторому образу $\Phi_{M}$ целочисленного когомологического класса при естественном гомоморфизме $H^{2}(M, \mathbb{Z})$ в $H_{d R}^{2}(\mathbb{C}) \simeq H^{2}(\mathbb{C})$. Многообразиями Ходжа являются $\mathbb{C}^{n}$ и $\mathbb{C} P^{n}$. Так как всякое подмногообразие ходжева многообразия является ходжевым, все естественным образом появляющиеся кэлеровы многообразия являются ходжевыми. Под хорошим покрытием понимается такое локально конечное открытое покрытие $\left\{U_{\alpha}\right\}$, что все элементы покрытия и их конечные пересечения являются стягиваемыми множествами. Для любой кэлеровой формы $\Phi_{M}$ многообразия $M$ ее образ при изоморфизме де Рама $H_{d R}^{2} \mapsto H^{2}(\mathbb{C})$ всегда можно представить вещественным 2-коциклом $x=\left(x_{\alpha \beta \gamma}\right)$ хорошего покрытия $\mathcal{U}=\left\{U_{\alpha}\right\}$. Этот коцикл определяется [9, с. 83-85] следуюшими условиями:

$$
\begin{gathered}
x_{\alpha \beta \gamma}=F_{\alpha \beta}+F_{\beta \gamma}+F_{\gamma \alpha}, \\
\operatorname{Im} F_{\alpha \beta}=F_{\beta}-F_{\alpha}, \\
-2 i \partial \bar{\partial} F_{\alpha}=\Phi_{\alpha}=\Phi_{M} \mid U_{\alpha},
\end{gathered}
$$

где $F_{\alpha \beta}$ - голоморфные функции, определенные на $U_{\alpha} \cap U_{\beta}$, а $F_{\alpha}$ вещественные функции на $U_{\alpha}$ (кэлеровы потенциалы). Пусть дано многообразие Ходжа $M, \Phi_{M}$ и некоторая целая, т.е. кратная образу целочисленного неделимого класса при изоморфизме де Рама, форма $\Omega=\frac{1}{m} \Phi_{M}$, где $m \in \mathbb{Z}$.

Рассмотрим экспоненциальную последовательность

$$
0 \mapsto \mathbb{Z} \mapsto \mathcal{O} \stackrel{2 \pi i \exp }{\longmapsto} \mathcal{O}^{*} \mapsto 0
$$

где $\mathcal{O}$ и $\mathcal{O}^{*}$ - это пучки ростков: голоморфных функций на $M$ и голоморфньх функций, необращающихся в 0 . Через $\mathbb{Z}$ обозначен постоянньй целочисленньй пучок. С одной стороны, хорошо известно, что

$$
\operatorname{Pic}(M) \simeq H^{1}\left(\mathcal{O}^{*}\right) \stackrel{c}{\longmapsto} H^{2}(\mathbb{Z})
$$


группа Пикара голоморфных линейных расслоений, изоморфная 1-й групе когомологий с коэффициентами в пучке $\mathcal{O}^{*}$ характеристическим отображением $c$, порожденным экспоненциальной последовательностью, отображается во 2-ю группу целочисленных когомологий. Линейное эрмитово расслоение $L, h$ над $M$ будем назьвать $n p u c o е д u-$ ненным к форме $\Omega$, если форма кривизны эрмитовой связности совпадает с формой $\Omega$. Как показано [9, с. 79-80], с точностью до изоморфизма де Рама характеристический класс $L$, определяемьй с помощью связывающего гомоморфизма для экспоненциальной последовательности, совпадает с формой кривизны; $c(L)=\Omega$.

Лемма 4. Если $\Phi_{м}$ форма Ходжса многообразия $M$, то существует линейное эрмитово расслоении $L, h$, присоединенное $\kappa$ форме $\Phi_{M}$, у которого локально координатные представления әрмитова скалярного произведения $h_{\alpha}$ относительно некоторого атласа $\mathcal{U}=\left\{U_{\alpha}\right\}$ имеют вид

$$
h_{\alpha}=\exp \left(-4 \pi F_{\alpha}\right) \text {, }
$$

где $F_{\alpha}$ - это вещественные кәлеровы потенииалы для $\Phi_{M}$ на $U_{\alpha}$.

ДоказАтЕльство. Применяя изоморфизм де Рама, определенный формулами (4.1)-(4.3), в случае ходжевой формы $\Phi_{M}$, можно заключить, что в этом случае 2-коцикл $x=\left\{x_{\alpha \beta \gamma}\right\}$ когомологичен целочисленныму 2-коциклу $c=\left\{c_{\alpha \beta \gamma}\right\}$, т.е. существуют такие комплексные числа $P_{\alpha \beta}$, что

$$
c_{\alpha \beta \gamma}=x_{\alpha \beta \gamma}+P_{\alpha \beta}+P_{\beta \gamma}+P_{\gamma \alpha}
$$

для любых $\alpha, \beta, \gamma$ таких, что $U_{\alpha} \cap U_{\beta} \cap U_{\gamma} \neq \varnothing$. Покрытие при этом, если необходимо, заменяется более тонким. Как известно [9, с. 83-85], функции $f_{\alpha}^{\beta}$, определенные формулой

$$
\begin{gathered}
f_{\alpha}^{\beta}=\exp \left(2 \pi i Q_{\alpha \beta}\right): U_{\alpha} \cap U_{\beta} \mapsto \mathbb{C}^{*}, \\
Q_{\alpha \beta}=F_{\alpha \beta}+P_{\alpha \beta},
\end{gathered}
$$

являются функциями перехода голоморфного линейного расслоения, характеристический класс $c(L)$ которого равен $\Phi_{M}$.

Обозначим через $\mathbb{Z}$ постоянньй $\mathbb{Z}$-пучок, а через $\mathcal{O}^{*}$ - пучок ростков необращающихся в нуль голоморфных функций на $M$. Хорошо известно, например, из формулы (11) 22 главы I в [10] то достаточно очевидное обстоятельство, что экспоненциальная последовательность является короткой точной последовательностью для любого комплексного многообразия. По построению голоморфного линейного расслоения $L$ над $M$ его характеристический класс $c(L)$, определенньй из экспоненциальной последовательности, совпадает с целочисленным когомологическим классом $c$ из формулы (4.5). При этом, конечно, $c(L)$ отождествляется со своим образом в комплексных когомологиях $H^{2}(\mathbb{C})$ при отображении в когомологиях индуцированным естественным вложением $\mathbb{Z} \mapsto \mathbb{C}$.

Переходя к построению эрмитова скалярного произведения $h$ в $L$, следует прежде всего отметить, что комплексные числа $P_{\alpha \beta}$ в (4.5) можно выбрать чисто вещественными. Если бы это было не так, то можно было бы сделать замену $P_{\alpha \beta}:=\operatorname{Re} P_{\alpha \beta}$, отбросив мнимую часть. Так как $x$ вещественный коцикл, значение $c_{\alpha \beta \gamma}$, определенное формулой (4.5), после такой замены не изменится. Эрмитово скалярное произведение $h$ 
определим локально координатными представлениями $h_{\alpha}$ относительно использованного вьшше атласа $\mathcal{U}$ :

$$
h_{\alpha}=\exp \left(-4 \pi F_{\alpha}\right) \text {, }
$$

где функции кэлерова потенциала $F_{\alpha}$ те же, что и прежде. Корректность так определенного эрмитова скалярного произведения следует из сравнения крайних частей в цепи равенств

$$
h_{\alpha}\left|f_{\alpha}^{\beta}\right|^{2}=h_{\alpha} \exp \left(-4 \pi \operatorname{Im} F_{\alpha \beta}\right)=h_{\beta},
$$

при получении которых использовалась формула (4.2) и отсутствие мнимой части у величин $P_{\alpha \beta}$.

Лемма 5. Если $\Phi_{M}$ форма Ходжа многообразия $M$, то существует линейное әрмитово расслоении $L^{*}, h^{*}$, присоединенное к форме $-\Phi_{M}$, у которого локально координатные представления эрмитова скалярного произведения $h_{\alpha}^{*}$ относительно некоторого атласа $\mathcal{U}=\left\{U_{\alpha}\right\}$ имеют вид

$$
h_{\alpha}^{*}=\exp \left(4 \pi F_{\alpha}\right) \text {, }
$$

где $F_{\alpha}$ - это вещественные кәлеровы потенциалы для $\Phi_{M}$ на $U_{\alpha}$.

ДокаЗАтЕльство. Характеристическое отображение $c$ из (4.4) является групповым гомоморфизмом относительно операции тензорного умножения расслоений в группе $\operatorname{Pic}(M)$ и сложения когомологических классов в $H^{2}(M, \mathbb{Z})$. Поэтому из формулировки леммы следует, что голоморфное линейное расслоение $L^{*}$ - двойственное к расслоению $L$ из леммы 4. Поэтому функциии перехода $\varphi_{\alpha}^{\beta}=\exp \left(-2 \pi i Q_{\alpha \beta}\right)$ расслоения $L^{*}$ для атласа $\mathcal{U}$, определяемого формулами (4.1)-(4.3), являются обратными к голоморфным функциям перехода расслоения $L$ из формулы (4.6). Определенное в формулировке леммы локальное представление эрмитова скалярного произведения корректно, потому что вместо формулы (4.8) вьполняются следуюшие равенства.

$$
h_{\alpha}^{*}\left|\varphi_{\alpha}^{\beta}\right|^{2}=h_{\alpha}^{*} \exp \left(4 \pi \operatorname{Im} F_{\alpha \beta}\right)=h_{\beta}^{*}
$$

Теорема 5. Каждое ходжево многообразие $M$ может служить базой скрещенного кәлерова расслоения Е. В качестве стандартного слоя мохсет выступать риманова поверхность, голоморфно әквивалентная одной из следующих поверхностей:

а) единичному кругу с выколотым иентром $B_{1}^{*}$;

б) комплексной прямой с выколотой точкой $\mathbb{C}^{*}$;

в) всей комплексной прямой $\mathbb{C}$;

г) $B_{1}-$ единичному кругу в $\mathbb{C}$;

д) $\mathbb{C} P^{1}$ - сфере Римана;

е) кольиу $K=\{w \in \mathbb{C} \mid A<w<B\}$, причем $0<A<B<+\infty$.

Причем в случаях а), б) и е) показатель конформности $f$ всюду невырожсднный, в случаях в) иг) имеет ровно одно әкстремальное значение, а в случае д) - имеет ровно два әкстремальных значения. 
ДокАЗАТЕЛЬСТво. Пусть $\Phi_{M}$ форма Ходжа многообразия $M$. Воспользуемся леммами 4,5 . Пусть линейное эрмитово расслоение $L, h$ является присоединенньг к форме $\Omega=\Phi_{M}$, a $L^{*}, h^{*}-$ к форме $\Omega=-\Phi_{M}$. Через $\|\Xi\|$ будем обозначать норму вектора $\Xi$ как в первом, так и во втором расслоениях. Имеем

$$
h_{\alpha} w_{\alpha} \bar{w}_{\alpha}=\|\Xi\|^{2} \quad \text { или } \quad h_{\alpha}^{*} w_{\alpha} \bar{w}_{\alpha}=\|\Xi\|^{2} .
$$

Формулами (4.10), (4.11) определим на $E\left(\lambda=1\right.$ или $=-1$ для $L$ или $L^{*}$ соответственно) форму типа $(1,1)$, где $\nu$ - это проекция в расслоении $L$ или $L^{*}$. Имеем

$$
\begin{gathered}
p^{2}(s)=p_{c}^{2}-2 \lambda \int_{C}^{s} \sigma\left(\frac{d l}{d \sigma}\right)^{2} d \sigma>0, \\
\Phi=-\frac{2 i}{\pi} \partial l(s) \wedge \bar{\partial} l(s)+p^{2}(s) \nu^{*} \Phi_{M}, \quad \text { где } \quad s=\|\Xi\| .
\end{gathered}
$$

Пусть $l=l(s)$ гладкая невырожденная на промежутке от $A$ до $B$ функция $(0 \leqslant A<B \leqslant$ $+\infty)$ такая, что выполняется неравенство в (4.10) для некоторого $C$ из промежутка от $A$ до $B$. Пусть равенством в (4.10) определена положительная функция $p(s)$ для $\lambda=1$ или для $\lambda=-1$. Пусть $E$ - подрасслоение в $L$ или в $L^{*}$, состоящее из векторов, норма которых принадлежит промежутку от $A$ до $B$. Сопоставляя выражение, полученное для $s$ из формул (4.11), (4.9) при помощи лемм 4, 5, с выражением в (3.11), приходим к вьводу, что форма $\Phi$, заданная формулой $(4.11)$, в каждой порции над $U_{\alpha}$ совпадает с формой $\Phi$ из примера 2. На основании примера 2 можно заключить, что расслоение $E$, оснащенное формой $\Phi$, является скрещенным кэлеровым расслоением. Выбором параметров $A, B$ и функции $l(s)$ можно реализовать каждый пункт теоремы, кроме д). Так, для функции $l(s)$, определенной на $(0 ; B)$, где $0<B \leqslant+\infty$, реализуются пункты а), б). Для $l(s)$, определенной на $[0 ; B)$, реализуются пункты в), г). Для случая е) параметры выбираются так: $0<A<B<+\infty$. Выбор указанных параметров и функции $l(s)$, очевидно, возможен для $\lambda= \pm 1$.

д) Этот случай требует отдельного рассмотрения. Пусть $l(s)$ произвольная гладкая невырожденная (в 0 справа) функция, определенная на $[0 ;+\infty)$. Причем $l(s)$ выберем так, чтобы порядок производной $l^{\prime}(s)$ на бесконечности относительно величины $1 / s$ был равен 2. Это условие обеспечивает, в частности, сходимость несобственного интеграла в (4.12):

$$
\begin{gathered}
\frac{d l}{d s} \sim \frac{k}{s^{2}}(s \rightarrow+\infty), \quad 2 \int_{0}^{+\infty} \sigma\left(\frac{d l}{d \sigma}\right)^{2} d \sigma=p_{0}^{2}-p_{\infty}^{2}>0 ; \\
p^{2}(s)=p_{1}^{2}-2 \int_{1}^{s} \sigma\left(\frac{d l}{d \sigma}\right)^{2} d \sigma>0 .
\end{gathered}
$$

Выберем произвольное положительное число $p_{1}$ таким, чтобы вьполнялось неравенство в (4.13). Тогда пара равенств: (4.12) и $p_{1}^{2}=\left(p_{0}^{2}+p_{\infty}^{2}\right) / 2,-$ однозначно определяют значение $p_{0}\left(0<p_{1}<p_{0}\right)$ положительной функции $p(s)$, определяемой равенством (4.13). Причем $p_{0}=p(0)$, а $p_{\infty}$ есть предел этой функции при $s \rightarrow+\infty, p_{\infty}>0$. Например, функция $l(s)=\cos (\pi /(4 \sqrt{s+1}))$ удовлетворяет условию невырожденности и для нее вьполняется эквивалентность из (4.12). 
Теперь перейдем к построению расслоения $E$, слой которого сфера. Оно будет получено, образно говоря, путем заклеивания расслоения $L$ в бесконечности. Пусть $L \oplus L^{*}-$ это прямая сумма голоморфных линейных расслоений, определяемая традиционным образом, как, например, в [10, с. 69]. В обоих слагаемых кэлерова структура определяется с помощью формулы (4.11), но функции $l(s)$ и $p(s)$ для первого расслоения, определенные вьше формулами (4.12), (4.13), для второго экземпляра расслоения определяются иначе. Обозначим их с волной вверху:

$$
\tilde{l}(r)=l\left(\frac{1}{r}\right), \quad \tilde{p}^{2}(r)=p_{1}^{2}+2 \int_{1}^{r} \rho\left(\frac{d \tilde{l}}{d \rho}\right)^{2} d \rho .
$$

Эквивалентность в (4.12) означает невырожденность $\tilde{l}$ в нуле. Рассмотрим отображение инверсии между указанными расслоениями с вырезанными нулевыми сечениями: $L_{0}$ и $L_{0}^{*}$. В ранее введенных координатах эта инверсия определяется так: $\left(z ; w_{\alpha}\right) \mapsto$ $\left(z ; w_{\alpha}^{-1}\right)$. Из формул (4.9) и лемм 4,5 вытекает, что нормы векторов при таком преобразовании взаимно обратны. Так как функции $l$ и $p$ переходят в функции $\tilde{l}$ и $\tilde{p}$ при замене переменных $r$ на $1 / s$, инверсия осушествляет кэлеров изоморфизм между двумя кэлеровьми многообразиями $L_{0}$ и $L_{0}^{*}$. Выберем в качестве функции склейки инверсное отображение. Полученное после склейки многообразие обозначим через $E$. Из построения ясно, что $E$ имеет своим слоем $S^{2}$ и удовлетворяет всем остальным требованиям теоремы.

Расслоенное пространство назьвается полным, если оно является полным как риманово многообразие.

Теорема 6. Каждое полное ходжево многообразие $M$ может служить базой полного скрещенного кәлерова расслоения Е. В качестве стандартного слоя полного расслоения Е может выступать риманова поверхность, голоморфно әквивалентная одной из следующих поверхностей:

а) единичному кругу с выколотым иентром $B_{1}^{*}$;

б) комплексной прямой с выколотой точкой $\mathbb{C}^{*}$;

в) всей комплексной прямой $\mathbb{C}$;

г) $B_{1}-$ единичному кругу в $\mathbb{C}$;

д) $\mathbb{C} P^{1}$ - сфере Римана, при этом $f$ имеет ровно два әкстремальных значения. Причем в случаях а) и б) показатель конформности $f$ всюду невырожденный, в случаях в) иг) имеет ровно одно әкстремальное значение, а в случае д) имеет ровно два әкстремальных значения.

ДокАЗАТЕЛЬСтво. Будем применять теорему 5, выбирая специальньм образом функцию $l(s)$. Для того чтобы построенное кэлерово расслоение $E$ оказалось полным, необходимо выбирать функцию $l$, удовлетворяющей дополнительному свойству:

1) для пунктов a), б) образ функции $\operatorname{Im} l=(-\infty ;+\infty)$;

2) для пунктов в), г) $A=0$ и $\operatorname{Im} l=[0 ;+\infty)$.

Эти дополнительные условия на $l(s)$ должны вьполняться одновременно с прежним условием справедливости неравенства в (4.10) для любых $s$. Это означает, что несобственньй (в силу дополнительных условий) интеграл в неравенстве

$$
\int_{A}^{B} \sigma\left(\frac{d l}{d \sigma}\right)^{2} d \sigma>0
$$


при $\lambda=1$ должен сходится на верхнем пределе, а при $\lambda=-1$ - на нижнем. При таком выборе $l(s)$ слой $S$ расслоения $E$ является полньм римановым многообразием. В пункте д) слой является полньм римановым многообразием по построению, более того, здесь слой - компакт. Случай а) реализуется, например, для функции $l(s)$, заданной равенством

$$
l(s)=\ln \ln (s / A):(A ;+\infty) \mapsto \mathbb{R}
$$

для $\lambda=1$. Очевидно, что кольцо с внешним радиусом, равным бесконечности, голоморфно эквивалентно кругу с выколотьм центром. Случай б) реализуется, например, для функции $l(s)$, заданной равенством

$$
l(s)=\ln \ln (1+s):(0 ;+\infty) \mapsto \mathbb{R}
$$

для $\lambda=1$. Случай в) реализуется, например, для функции $l(s)$, заданной равенством

$$
l(s)=\ln (1+\ln (1+s)):[0 ;+\infty) \mapsto \mathbb{R}
$$

для $\lambda=1$. Случай г) реализуется, например, для функции $l(s)$, заданной равенством

$$
l(s)=\operatorname{tg} \frac{\pi s}{2 B}:[0 ; B) \mapsto \mathbb{R}
$$

для $\lambda=-1$.

Итак, во всех пунктах а)-д) построено скрещенное кэлерово расслоение $E$ с полньм слоем. Порция $E$ над некоторой окрестностью любой точки изоморфна расслоению из примера 2. В (3.15) имеется выражение для римановой метрики $g$, ассоциированной с $\Phi$. Отображение, сопоставляющее вектору $\Xi$ вещественное число $l(\|\Xi\|) / \sqrt{2 \pi}$, является римановой субмерсией из $E$ на $\mathbb{R}$ относительно канонической метрики прямой. Рассмотрим произвольную фундаментальную последовательность векторов $\Xi_{k}$ в $E$ относительно дистанции, порожденной $g$. Числовая последовательность $l\left(\|\Xi\|_{k}\right)$ также является фундаментальной и, следовательно, ограниченной. Так как $p^{2}(l)$ гладкая функция от $l$, существуют такие положительные числа $c$ и $r$, что неравенства

$$
r \geqslant p^{2}(\|\Xi\|) \geqslant c
$$

выполняются при $\Xi=\Xi_{k} \forall k$. Пусть замкнутоев $E$ подмножество $Q$ состоит из векторов $\Xi$, норма которых удовлетворяет неравенствам в (4.19), т.е. $\left\{\Xi_{k}\right\} \subset Q$. Рассмотрим риманову метрику на $Q$, определенную формулой

$$
q=\frac{1}{2 \pi}\left(d l^{2}+d^{c} l^{2}\right)+c \cdot \nu^{*} g_{M} .
$$

Из формулы (3.15) и второго неравенства в (4.19) следует, что $q \leqslant g$, поэтому $\Xi_{k}$ является фундаментальной последовательностью и относительно дистанщии, порожденной $q$. Из первого неравенства в (4.19) следует, что спроектированная на базу последовательность $\nu_{*} \Xi_{k}$ является фундаментальной последовательностью в $M$ относительно дистанции, порожденной $g_{M}$. Из полноты базы следует, что при $k \rightarrow \infty \nu_{*} \Xi_{k} \rightarrow z \in U$, где $U$ - некоторое замкнутое множество базы, которое допускает тривиализацию. Обозначим $P=Q \cap \nu^{-1}(U)$. Риманово многообразие $(P, q)$ является прямым произведением полных римановых многообразий: замкнутого подмногобразия полного риманова пространства $S$ и базы с римановой метрикой $c g_{M}$. Поэтому $(P, q)$ также является полным римановым многообразием и относительно дистанции, порожденной $q$, рассматриваемая последовательность сходится. Из первого неравенства в (4.19) следует, что $g \leqslant r \cdot q$ и последовательность $\Xi_{k}$ сходится и относительно дистанции, порожденной $g$. Таким образом, $E$ является полным кэлеровым многообразием. 
ЗАмЕчАниЕ. Формулой (4.15) в расслоении $E$ определяется кэлерова метрика, слоями которой служат псевдосферы Бельтрами кривизны $-2 \pi$.

\section{СПИСОК ЦИТИРОВАННОЙ ЛИТЕРАТУРЫ}

[1] Hermann R. A sufficient condition that a mapping of Riemannian manifolds be a fiber bundle // Proc. Amer. Math. Soc. 1960. V. 11. № 2. P. 236-242.

[2] Окрут С.И. Скрещенное произведение в кэлеровой геометрии // Матем. физика, анализ, геометрия. 1997. Т. 4. № $1 / 2$.

[3] Кобаяси Ш., Номидзу К. Основы дифференциальной геометрии. В 2-х т. М.: Наука, 1981.

[4] Бессе А. Многообразия Эйнштейна. В 2-х т. М.: Мир, 1990.

[5] Molino P. Riemannian Foliations. Boston: Birkhäuser, 1988.

[6] Tonder Ph. Foliations on Riemannian manifolds. N. Y.: Springer-Verlag, 1988.

[7] Gray A. Pseudo-Riemannian almost product manifolds and submersions // J. Math. Mech. 1967. V. 16. № 7. P. 715-737.

[8] Nomizu K., Ozeki H. The existence of complete Riemannian metrics // Proc. Amer. Math. Soc. 1961. V. 12. №6. P. 889-891.

[9] Чжэнь Шэн-шэнь Комплексные многообразия. М.: ИЛ., 1961.

[10] Хирцебрух $\Phi$. Топологические методы в алгебраической геометрии. М.: Мир, 1973.

Харьковский государственный университет

Поступило

21.05.98 